\section{Cobalt, and Sheep Diseases}

IN New Zealand, Askew and his co-workers have shown that cobalt plays a vital part in the healthy life of sheep and that small supplements of cobalt salts will cure sheep suffering from a type of anæmia. Sheep on Dartmoor are liable to a disease very similar in symptoms to those described by the New Zealand workers, and the usual practice of farmers is to send their flocks to lowland pastures in the autumn for two or three months.

Analyses of the cobalt content of the soils from moorland pastures on which sheep suffer and from lowland pastures on which sheep have been known to recover give striking differences which are statistically significant :

Moorland soils on which sheep suffer have a mean cobalt contents of 3.9 parts per million; lowland soils on which sheep recover, $16 \cdot 7$ parts per million.

The figure for the sickness soils is higher than that given by Kidson ${ }^{1}$ for corresponding soils in New Zealand, where it was found that sickness was associated with soils having a cobalt content of two parts per million. In the Dartmoor district this figure would be about four parts per million, although occasionally samples have a slightly higher content.

Analyses of pastures show that those in the sickness areas have a mean content of 0.20 part per million cobalt and those in the recovery areas 0.45 part per million.

An examination of soil and pasture composition is in progress, and the relationship of these to the incidence of disease is being studied.

\section{The Laboratory, \\ Dartington Hall, \\ Totnes, S. Devon. August 13. \\ ${ }^{1}$ Kidson, N.Z.J. Sci. and Tech., 18, 694 (1937). \\ Effect of Occluded Hydrogen on the Rigidity of Metals}

AN investigation of the above effect was prompted by an inquiry into the nature of the earth's interior. The existence of a very definite core of radius half that of the earth seems established beyond reasonable doubt by seismology. The nature of this core is puzzling. Tidal phenomena and the Eulerian nutation demand that this core be less rigid than the crust. The noticeable absence of the shear wave on earthquake records of quakes sufficiently distant for the waves to pass through the core (and this, despite the presence of very pronounced compressional waves of the same quake on the record), was taken to mean that the core could not transmit a shear wave. A liquid core was therefore quite generally held to fit in with all known phenomena. However, during the past few years several seismic investigators, after exhaustive study of records, feel reasonably sure that the shear wave does pass through the core, but with greatly diminished energy. This would seem to imply either that a fluid under high pressure can transmit a shear wave, or that the core of the earth is a solid of very low rigidity. The idea suggested itself that a solid solution might satisfy this picture of a solid of low rigidity.

To test this, a solid solution of hydrogen in palladium was experimented with. The first question proposed was, "Does the occluded hydrogen affect the rigidity of the palladium ?" The purpose of this communication is to state that apparently it does. The rigidity was measured by means of a torsion pendulum. A small magnetized needle was suspended by a palladium wire half a millimeter in diameter. The needle was placed in an oscillating magnetic field of variable frequency. The period of the pendulum was obtained by measuring the frequency of the oscillator at resonance, which was found to be quite sharp. In the formula $\mu=8 \pi I l / \mathrm{r}^{4} T^{2} l, r$ and $T$ were determined before and after occlusion, which was produced by electrolysis. The results were quite erratic, some of the causes of which will be gone into later. However, there was a noticeable change produced in the rigidity of the metal, namely a decrease of rigidity with increasing occlusion. Some twenty different wires were tried and the change in rigidity measured when the wire was saturated with hydrogen. At first fairly long lengths of wire were used, but eventually it was found convenient to use short suspensions about $2 \cdot 5 \mathrm{~cm}$. long.

The average decrease in rigidity on saturation was found to be 13.7 per cent. The experiments were carried out in vacuo, in hydrogen and in air, but beyond a slight change in amplitude of the pendulum, no measurable change in period was noticed in the three cases. The best absolute value of the coefficient of rigidity of palladium into which no excess hydrogen has been forced was found to be $4 \cdot 257 \pm 0 \cdot 030 \times 10^{11}$. All palladium normally contains a certain amount of hydrogen apparently, and if this be driven off by passing a current through the wire, the above figure for the rigidity will be at least 12 per cent higher.

Starting with a piece of uncharged wire, a current of 10 amp. was passed through for a few seconds, heating the wire to redness. The rigidity increased $1 \cdot 7$ per cent. The current was again passed through for about a minute and the rigidity increased a further 8.4 per cent. The wire was then charged with about 400 times its volume of hydrogen. The rigidity decreased $16 \cdot 6$ per cent. Current was again passed through to drive out the hydrogen, this time for several minutes. The rigidity increased 22.8 per cent. The wire was again charged with about 450 times its volume of hydrogen and the rigidity decreased $21 \cdot 0$ per cent. It seems fairly certain, therefore, that the occluded hydrogen causes a decrease in the rigidity of the metal. The investigation is being continued further at Fordham and New York Universities jointly.

Note added in proof: The work of K. R. Koch in this field has not been overlooked, but will be discussed later.

$$
\begin{gathered}
\text { Fordham University, } \\
\text { New York City. } \\
\text { June } 28 .
\end{gathered}
$$

\section{Kinetics of Catalysed Polymerization of Styrene}

INVESTIGATIONS of polymerization kinetics have often required rather cumbersome experimental methods, but Hammick and Langrish ${ }^{1}$ have recently applied a convenient bromination method for estimating cyclopentadiene and indene to a study of the polymerization rates of these compounds in carbon tetrachloride. We have been using, for some time, a very similar method to follow the kinetics of the polymerization of styrene, catalysed by anhydrous stannic chloride, in carbon tetrachloride and chloroform solutions at $25^{\circ} \mathrm{C}$. The amount of residual monomeric styrene in the reaction mixture is determined, at known intervals of time, by quantitative bromine addition to the double bond, using an excess of bromine which is estimated by titration in the usual way. The accuracy of the analysis (better 